\title{
Evaluación en Ciencias Económicas. El caso "Costos para la Gestión". Registro de intervención académica
}

\author{
Evaluation in Economic Sciences. The case "Costs for Management". \\ Academic Intervention Record
}

\section{Romina Saullo}

https://orcid.org/0000-0001-9566-5483

rsaullo@hotmail.com

Facultad de Ciencias Económicas | UNLP | Argentina

\section{RESUMEN}

La presente intervención se basa en un ejercicio de reflexión acerca de las prácticas evaluativas en la materia "Costos para la Gestión", cuyo curriculum si bien pensado para que los espacios teóricos y prácticos y las instancias de evaluación respectivas se sucedan articuladamente, lo cierto es que una vez cerrada y aprobada la cursada práctica, los estudiantes dejan pasar un tiempo nada despreciable antes de rendir el examen final, lo que los coloca en una situación de desventaja respecto de los que rinden el examen final inmediatamente, es decir, en las primeras instancias. Se puede afirmar que existe una relación directa entre la distancia temporal entre cursada y examen y el desempeño poco satisfactorio en el mismo. Esta situación hizo que surgiera la inquietud y la necesidad de revisar la propuesta metodológica, resultando en la formulación de cursadas teórico prácticas bajo el régimen de promoción sin examen final. Este trabajo documenta la experiencia.

\section{ABSTRACT}

This work is based on an exercise of thought and deliberation about current evaluation practices in the subject "Costs for Management". Although the curriculum is designed so that the theoretical and practical spaces and the respective evaluation instances follow each other in an articulated way, the truth is that, once the course ends its practical classes, the students allow a significant time to go by before applying for the final exam, placing them in an actual disadvantageous position, compared to those who take the final exam immediately, that is, in the first available instances. It can be said that there is a direct relationship between the lapse of time between the course and the exam date and a below- standard performance in the latter. This situation led to the concern towards the urgent need to review the methodological approach regarding teaching and evaluation practices. This process resulted in the formulation of an academic offer that includes both theoretical and practical aspects at the same time, under a special regime that implies passing the course without any final exam. This document keeps record of that experience.

PALABRAS CLAVE

Evaluación, Intervención, Ciencias Económicas

KEY WORDS

Evaluation, Intervention, Economic Sciences 


\section{INTRODUCCIÓN}

La materia "Costos para la Gestión", de las carreras de Licenciado en Administración y de Contador Público, contiene una carga horaria obligatoria de práctica y una teórica de asistencia optativa. La evaluación se efectúa en dos instancias: los parciales (escritos y de contenido práctico) y el examen final que es oral y abarca todo el contenido teórico de la materia.

Si bien el curriculum fue originalmente pensado para que los espacios teóricos y prácticos y las instancias de evaluación respectivas se sucedan articuladamente, lo cierto es que una vez cerrada y aprobada la cursada práctica, los estudiantes dejan transcurrir un tiempo considerable antes de rendir el examen final, impactando negativamente en los resultados de la evaluación. Lo anterior ameritó la revisión de la propuesta metodológica de enseñanza y evaluación.

\section{JUSTIFICACIÓN Y CONTEXTO}

Ser evaluado implica someterse al juicio de otro y esa discrecionalidad conlleva la posibilidad de un desenlace desfavorable, por lo que pareciera existir un consenso en que dicho problema se resolvería garantizando transparencia y objetividad en los aspectos metodológicos.

"Qué, cómo y cuándo evaluar", parecieran ser las preguntas más difíciles de responder y muchas veces evaluar se traduce en la exigencia administrativa de tener que "poner una nota", una actividad despegada del aprendizaje y del sujeto que aprende.

\section{"Qué, cómo y cuándo evaluar", parecieran ser las preguntas más difíciles de responder y muchas veces evaluar se traduce en la exigencia administrativa de tener que "poner una nota", una ac- tividad despegada del aprendizaje y del sujeto que aprende.}

En este marco de revisión crítica de la evaluación, la presente intervención persigue en principio reducir los tiempos que transcurren entre la finalización de la cursada y su acreditación, alterando las prácticas de 
enseñanza y evaluación, promoviendo al mismo tiempo una mejora en el vínculo entre docentes y alumnos.

Dado que la factibilidad de implementación descansa principalmente en el contexto, es importante considerar que la Facultad de Ciencias Económicas es una unidad académica de notable eficiencia en la gestión, que acompaña el constante crecimiento de la matrícula ${ }^{1}$ con reformas edilicias e inversión en equipamiento. No obstante, si bien cuenta con personal muy especializado ${ }^{2}$, al igual que en otras facultades de ciencias económicas, la mayoría de los profesores consideran a la docencia e investigación como complementarias a la actividad profesional, incluso como tarea no remunerada ${ }^{3}$.

A su vez, en el diseño de metodologías de evaluación, se observa una marcada tendencia reproductiva, es decir, la Facultad puede ser inclusiva y adaptarse espacialmente a una matrícula que no cesa de crecer, lo que parece acentuar lo impersonal de los procesos evaluativos.

En el caso bajo estudio, los exámenes parciales escritos reconstruyen ejercicios de las clases prácticas obligatorias, evaluando la capacidad del alumno para resolver problemas. Son pruebas objetivas, con un formato cerrado que busca una respuesta correcta (Biggs, 2006: 214). Si bien, se suele pensar que eximen al evaluador de la subjetividad del juicio, lo cierto es que el juicio se traslada de la puntuación al diseño de un "buen examen".

Por su parte, el examen final es una instancia oral, con una importante cuota de discrecionalidad, donde se evalúa no sólo el contenido teórico, sino también la exposición, el lenguaje, sus opiniones, etc.

Enfrentados a una encuesta sobre la evaluación ${ }^{4}$, el 67\% de los alumnos estuvo de acuerdo con la combinación de dos exámenes parciales escritos y uno final oral. Si bien esta alternativa resultó la mejor calificada en cuanto a su validez, la mayoría le otorgó un puntaje bajo en objetividad. No obstante ello, resulta interesante destacar que, a pesar de que los estudiantes valoran la objetividad, ninguno prefirió una prueba objetiva de tipo opción múltiple, a pesar de que se ofrecía como alternativa.

La cuestión de la objetividad implica una situación problemática per se, por lo que la búsqueda de transparencia en la calificación y las restricciones de espacio y tiempo, se traducen en metodologías de evaluación que favorecen el divorcio entre teoría y práctica. Tanto exámenes parciales como finales, se traducen en una "calificación" que refleja el grado de alcance de los objetivos lo que, en el primer caso, cumple con los requisitos de objetividad y verificabilidad, pero se torna discrecional cuando se busca aplicar la misma pretensión al examen final.

Tanto exámenes parciales como finales, se traducen en una "calificación" que refleja el grado de alcance de los objetivos lo que, en el primer caso, cumple con los requisitos de objetividad y verificabilidad, pero se torna discrecional cuando se busca aplicar la misma pretensión al examen final. 
Lo anterior, seguramente aboga por la prevalencia de los exámenes escritos, en desmedro de las instancias orales, con baja adhesión entre los estudiantes, en consonancia a su vez, con otras dificultades relacionadas con clases cada vez más numerosas, producto de los muy significativos y deseados procesos de democratización de la educación (Camilloni, 1998: 3), por lo que el diseño de las prácticas evaluativas vigentes responde al mismo tiempo a restricciones de espacio y tiempo, permitiendo examinar a cohortes enteras simultáneamente.

La presente intervención intentó reducir la dilación temporal entre la finalización de la cursada y la acreditación y mejorar la percepción instalada de falta de objetividad de los exámenes orales, a través de cursos bajo el régimen de promoción sin examen final ${ }^{5}$, donde el objetivo es evaluar el rendimiento durante el curso, tanto en los aspectos teóricos, como en los prácticos, trabajando con grupos de hasta 60 estudiantes, a los que se accede por promedio $y / o$ sorteo, con un requisito de asistencia del $80 \%$ y un mínimo de seis (6) puntos en las evaluaciones.

Abordando la cuestión de la evaluación, podemos pensar en dos acepciones del término: un enfoque procesual, según el cual, evaluar es formular un juicio sobre la actuación de un individuo; y un enfoque estructural, según el cual, el sentido de evaluación hace referencia a todo proceso orientado al conocimiento de la calidad del servicio educativo prestado (Angulo Rasco, 1994: 283). Más allá de la complejidad de cada una, en ambos casos estamos hablando de la formulación de un juicio.

Por su parte, los sistemas de calificación constituyen un componente esencial de los sistemas de evaluación, ejerciendo un fuerte efecto normativo sobre las acciones de enseñanza y aprendizaje y no permanecen estáticos, sino que deben acompañar las transformaciones de las prácticas, influyendo en las estrategias de enseñanza de los docentes y particularmente, en las estrategias de aprendizaje de los alumnos.

La idea que aquí se suscribe es la de evaluación continua, basado en el análisis e interpretación de las observaciones efectuadas por el docente respecto de las conductas, los procesos y las producciones de los alumnos en situaciones no puntuales de evaluación, no diferenciadas del aprendizaje.

\section{La idea que aquí se suscribe es la de evaluación continua, basa- do en el análisis e interpretación de las observaciones efectua- das por el docente respecto de las conductas, los procesos y las producciones de los alumnos en situaciones no puntuales de evaluación, no diferenciadas del aprendizaje}

Por lo tanto y más allá de las diversas opciones, es innegable la existencia de un aspecto enteramente personal, ya que subyace siempre una opinión que atraviesa la evaluación. Así, el afán de generar instrumentos de medición desprovistos de toda subjetividad, se torna estéril en la medida en que evaluar implica necesariamente un juicio. Enseñar exige buen juicio (Freire, 2004: 60) y lo que resulta bien cierto como 
cuando nos referimos a la evaluación.

Sin embargo, ese "buen juicio" se pone en práctica más en el diseño y en el recorte de los contenidos y que en la planificación de la evaluación, dado que se la suele imaginar y diseñar como a actividad separada de la enseñanza o como el cierre que sirve para "abrochar" las metas pedagógicas previamente trazadas (Carlino, 1999: 84). Es por eso, que muchos autores ponen énfasis en señalar el carácter permanente de la evaluación, como parte integrante del proceso de interacción que se desarrolla entre profesor y alumno, que puede aportar información a los estudiantes, acerca de sus propios aprendizajes, y a los profesores, acerca de los efectos de su enseñanza, incluso utilizarla como medio para autoevaluarse 6 .

La selección y decisión por el modelo, descubre las concepciones y también los ideales político sociales del docente. Existen dos grandes orientaciones: la técnica y la crítica progresista (Barraza Macías, 2010: 15). De acuerdo a la primera, la evaluación se define como prueba sumativa, situada al final del ciclo para comprobar hasta qué punto los estudiantes han aprendido bien lo que se supone que han aprendido (Biggs, 2006: 179), mientras que, la racionalidad crítica pone acento en el diálogo y la colaboración como recursos centrales para la construcción de medios y fines deseables para la educación, lo que implica necesariamente un mayor protagonismo de los agentes.

La evaluación en ciencias económicas ha recurrido tradicionalmente al uso de pruebas objetivas en un temprano afán tecnicista, buscando precisión, transparencia y objetividad, además de resolver las restricciones de espacio y tiempo y la necesidad de examinar simultáneamente a una cantidad creciente de estudiantes. La evaluación devino un acto final desprendido de las acciones propias de la enseñanza y el aprendizaje, con el objetivo de comprobar, constatar o verificar el cumplimiento de unos objetivos (Celman, 1998: 67). No obstante ello, en este trabajo se suscribe, en lo concerniente a la práctica educativa en general y a la evaluación en particular, a la orientación crítica asumiendo que los problemas no son obstáculos sino motores para la mejora y que la intervención implica promover la cooperación y el diálogo entre protagonistas.

en este trabajo se suscribe, en lo concerniente a la práctica educativa en general y a la evaluación en particular, a la orientación crítica asumiendo que los problemas no son obstáculos sino motores para la mejora y que la intervención implica promover la cooperación y el diálogo entre protagonistas

A su vez, se entiende el proceso de evaluación como una acción multidimensional y subjetiva (Hoffmann, 2010: 73), que se inicia al principio de un curso cuando profesor y alumnos entran en contacto, negocian los elementos del programa y asumen niveles de exigencia y trabajo individual y grupal, que serán revisados y "re-pactados" a lo largo del curso, al tiempo que se asume un enfoque cualitativo de la evaluación, entendiendo que los procesos de razonamiento en las 
ciencias sociales, son complejos y requieren estrategias de evaluación que necesariamente no pueden ser cuantificables como instrumentos objetivos de medición.

Por supuesto que en ciertos ámbitos la objetividad está sobrevaluada y la idea de que los alumnos le otorgan más crédito a una prueba objetiva cuenta con una adhesión respetable, que en conjunto con las restricciones de tiempo y espacio descriptas refuerza la búsqueda de instrumentos válidos y confiables que pudieran ser garantía para alcanzar la ansiada objetividad, al tiempo que respeta el uso eficiente de los recursos escasos. Sin embargo, las encuestas realizadas reflejan una postura bien clara de los estudiantes, que están a favor de la diferenciación, y de los procesos singulares de enseñanza y aprendizaje. Así vemos que valoran el ser parte del diseño y saber en qué condiciones serán evaluados.

En esta propuesta la enseñanza es entendida como un proceso continuo de negociación de significados, que supone cambios, y junto con la evaluación, son los pilares del proceso de transformación del conocimiento en un dispositivo pedagógico que los abarca y que evidencia ciertos principios ordenadores que pueden ser los mismos o diferentes.

\section{En esta propuesta la enseñanza es entendida como un proceso continuo de negociación de significados, que supone cambios, y junto con la evaluación, son los pilares del proceso de trans- formación del conocimiento en un dispositivo pedagógico que los abarca y que evidencia ciertos principios ordenadores que pueden ser los mismos o diferentes}

Esto genera cierta confusión en los estudiantes, que optan en general, por seguir en su aprendizaje, la dirección que les señala la modalidad de evaluación que saben que adoptará el profesor. De ahí la importancia del alineamiento efectivo de los principios ordenadores de ambas dimensiones, la enseñanza y la evaluación.

En ese sentido, se pretende estrechar el vínculo docente y alumno, como un vehículo para resolver la problemática planteada del desánimo a la hora de inscribirse para acreditar la materia, al tiempo que refuerza la defensa de la evaluación oral, como instancia singular y diferenciada. Esto no quiere decir que se constituye en una evaluación exclusiva, sino que es el final de un proceso formativo, que genera una retroalimentación permanente con el docente y una mejora continua de su práctica. En este punto conviene definir a la evaluación formativa como aquella cuyos resultados se utilizan con fines de retroinformación, que debe servir tanto para mejorar el aprendizaje como para mejorar la enseñanza, de la que es inseparable (Biggs, 2006: 179). La evaluación formativa es a su vez, diferenciada, porque implica procesos diferenciales de enseñanza, en la comprensión de que alumnos con diferentes perfiles pueden alcanzar igual dominio de las competencias buscadas (Bertoni-Poggi-Teobaldo, 1997, 94). 


\section{INTERVENCIÓN. VIABILIDAD Y ESCENARIO}

Las prácticas evaluativas vigentes de la materia datan de la puesta en vigencia del plan de estudios, en 1992 y si bien, se han introducido cambios en el programa, no está entre las posibilidades alterar la carga horaria ni otros requisitos de acreditación. La alternativa es la introducción de un programa nuevo, que establezca la convivencia de un curso por promoción con las características antes descriptas, con la cursada regular, ambos con clases teóricas los martes y prácticas los jueves, con franjas horarias de tres horas.

En ambos cursos, la propuesta supuso explicar y discutir cada martes el tema teórico correspondiente al trabajo práctico del jueves de la misma semana. Para el caso del curso "regular", la necesidad real de asistir a la clase teórica, para poder atravesar con éxito la práctica, dosifica la lectura de la bibliografía, traduciéndose luego en un beneficio tangible para el estudiante: la posibilidad de rendir el examen final inmediatamente, sin incurrir en una revisión exhaustiva de todo el contenido teórico, con el condimento adicional de no salirse de la órbita de la cotidianeidad con el docente, el cual de seguro arriba al final del curso con una idea calificada, con una evaluación cualitativa y formativa acerca del alumno.

Incluso, se ofreció a los estudiantes de la cohorte vigente, la posibilidad de elegir rendir su examen con el profesor a cargo de la comisión a la que asistieron. Esta opción debe ser parte del contrato didáctico al comienzo del curso, como un incentivo que favorezca la asistencia a las clases teóricas no obligatorias, al tiempo que permite a los estudiantes delinear sus estrategias y sus calendarios. Lo anterior les otorga a los alumnos un rol protagónico, al validar su derecho: a saber de antemano cómo serán evaluados, y así sentirse parte de ese proceso.

En ese sentido, la mayor articulación entre teoría y práctica, se traduce también en una mejor performance de los alumnos en los exámenes parciales, al capitalizar los contenidos teóricos a medida que avanza el curso y por otro lado, los docentes a la hora de evaluar, cuentan con un juicio previo de los estudiantes, ya que trabajar con un grupo más o menos constante, propicia una nueva dinámica áulica, abandonando un poco la tradicional clase magistral para dar lugar a metodologías más participativas, que permiten la individualización y evaluación como proceso continuo.

\section{PUESTA EN PRÁCTICA Y SEGUIMIENTO}

Implementar una intervención académica, requiere siempre, además de un plantel comprometido con la propuesta, condiciones institucionales y una voluntad política que legitime los procesos. Afortunadamente, la cátedra está inserta en un Departamento que promueve la mejora continua de los procesos de enseñanza y aprendizaje.

En relación al seguimiento de la experiencia, se pensó en indicadores que reflejen la reducción de la brecha temporal entre finalización de cur- 
sada y acreditación final de la materia, para los cursos regulares. El uso de indicadores está muy asociado a la orientación tecnicista, paradigma que obtuvo una adhesión temprana en las ciencias económicas, y que parece abatido por la necesidad de garantizar procesos de evaluación diferenciados, no obstante ello, no es prudente abandonar de inmediato dicha pretensión eficientista como modelo de evaluación sumativa, ya que la construcción de un indicador que evidencie por ejemplo el número de alumnos de la cohorte vigente que finalmente acreditan la materia en la instancia intervenida, brinda una idea del éxito del proyecto en términos netamente físicos.

La implementación finalmente se llevó a cabo en 2013, con tres cursos "especiales", garantizando igualmente la opción de "cursada regular" para el resto de los estudiantes, ampliándose al año siguiente a cinco cursos especiales. El cuadro siguiente resume los resultados de la iniciativa y la reducción de la cantidad de inscriptos en las mesas de finales.

COSTOS PARA LA GESTIÓN ESTADISTICAS DE ACREDITACIÓN

\begin{tabular}{|c|c|c|c|c|c|}
\hline EDICION & $\begin{array}{c}\text { INSCRIPTOS } \\
\text { Curso Especial }\end{array}$ & $\begin{array}{c}\text { ABANDONO } \\
\text { AUSENTES }\end{array}$ & $\begin{array}{c}\text { Alumnos } \\
\text { APROBADOS }\end{array}$ & IMPACTO & $\begin{array}{l}\text { INSCRIPTOS } \\
\text { Curso Regular }\end{array}$ \\
\hline 2014 & 232 & 21 & 181 & $78 \%$ & 219 \\
\hline 2015 & 237 & 16 & 191 & $81 \%$ & 222 \\
\hline 2016 & 211 & 22 & 162 & $77 \%$ & 235 \\
\hline 2017 & 212 & 18 & 156 & $74 \%$ & 238 \\
\hline TOTALES & 892 & 77 & 690 & $77 \%$ & 914 \\
\hline
\end{tabular}

Al cabo de cinco años, el impacto de la intervención en el problema disparador no resulta, en efecto, medible en términos objetivos, sin embargo, se puede afirmar que en la actualidad es difícil encontrar estudiantes que hayan esperado más de dos años para acreditar la materia. La posibilidad de acceder al curso especial, devino a su vez en un incentivo adicional, ya que es notable la cantidad de alumnos que, habiendo aprobado la cursada en años anteriores, optan por volver a transitar el espacio, bajo la nueva modalidad, lo que les ofrece la doble ventaja de contar con saberes previos al tiempo que los pone en diálogo con un contenido actualizado.

Finalmente, en cuanto a la comunicación de los resultados de la propuesta, partiendo de la idea de que toda innovación educativa debe ser socializada, ya que todo proceso de innovación es idiosincrásico y está contextualizado (Barraza Macías, 2010: 96), se optó por utilizar la revista institucional de la Facultad, que ofrece un espacio de opinión y comunicación de experiencias. 


\footnotetext{
${ }^{1}$ Para el 2013, la matrícula fue de 2135 ingresantes. (Fuente: Memoria de la FCE 2010-2014 https://www. econo.unlp.edu.ar/facultad/memoria_2010_2014_fce-2263)

2 Fuente: Departamento Personal de la FCE. Sistema de Legajo Docente (SILEG), junio 2012.

${ }^{3}$ El 84\% de los docentes tiene dedicación simple y el 60\% de los docentes investigadores pertenecen a la categoría inferior (V). https://www.econo.unlp.edu.ar/la_facultad_en_cifras/sintesis_indicadores-2314

${ }^{4}$ La encuesta se llevó a cabo para los cohortes 2009 a 2012 y solicitaba a los estudiantes calificar distintas opciones de evaluación en cuanto a su objetividad y validez (Biggs, 2006: 203)

${ }^{5}$ ORDENANZA 115 - Texto Ordenado (2/7/12) Expte.900-5645/12 - Modificada por Ordenanza № 162/12

${ }^{6}$ La evaluación es prioritariamente considerada desde el ángulo de la valoración de los aprendizajes de los alumnos, en su proceso y en sus resultados. Sin embargo, tiene funciones significativas para la valoración de la propuesta de enseñanza misma. En otras palabras, el proceso de aprendizaje desarrollado y sus resultados, no son sólo una consecuencia de los alumnos, sino un producto de la enseñanza misma. Ello implica potenciar la evaluación como estrategia permanente de mejora y perfeccionamiento de las propuestas de enseñanza. (Davini, 2008: 226). http://www.econo.unlp.edu.ar/uploads/docs/revista_institucional_fce
}

\section{BIBLIOGRAFIA}

Angulo Rasco, J. F. y Blanco García, N. (1994). “Teoría y desarrollo del currículum", Málaga: Aljibe

Barraza Macías, A. (2010). “Elaboración de Propuestas de Intervención Académica". Univ. Pedagógica de Durango.

Bertoni, A. y otros (1995). “Evaluación: nuevos significados para una práctica compleja". Kapelusz.

Biggs John B. (2006). “Calidad del aprendizaje universitario”. (Capítulos 8 y 9). Madrid: Narcea.

Bohoslavsky, R. (1971).“Psicopatología del vínculo profesor alumno: el profesor como agente socializante". Galerna.

Camilloni, A. (1998). "Sistemas de calificación y regímenes de promoción" y "La calidad de los programas de evaluación y de los instrumentos que lo integran" en Camilloni, A. y otros: "La evaluación de los aprendizajes en el debate didáctico contemporáneo". Paidós.

Candreva, A. y Morandi, G. (1999). “El curriculum universitario: entre la teoría y la práctica" en: García Santa María, M. T. Un curriculum de ciencias sociales para el siglo XXI: qué contenidos y para qué. España: Díada Editora.

Carlino, F. (1999). “La evaluación educacional. Historia, problemas y propuestas". Aique.

Celman, S. (1993). “La tensión teoría-práctica en la educación superior". Entre Ríos: UNER. 
Celman S. (2003). "Sujetos y objetos en la evaluación universitaria". Facultad de Cs. Humanas, UNSL.

Celman, S. (1998). “¿Es posible mejorar la evaluación y transformarla en herramienta de conocimiento?" En Camilloni, A. y otros: "La evaluación de los aprendizajes en el debate didáctico contemporáneo". Paidós.

Davini, M. C., (2008). “Métodos de Enseñanza, didáctica general para maestros y profesores" Santillana.

Díaz Barriga, A. (1994). "Docente y Programa. Lo institucional y lo didáctico". Aique.

Díaz Barriga,A. (2000). “El examen". México: UNAM.

García, C. M. (1998). "El proyecto Docente: una ocasión para aprender" en: "Didáctica Universitaria" de Ana García-Valcárcel Muñoz-Repiso (coord.) Madrid: La Muralla.

Gimeno Sacristán, J. (1992). “Diseño del curriculum, diseño de la enseñanza. El papel de los profesores" en: Gimeno Sacristán, J. y Pérez Gómez, A. “Comprender y transformar la enseñanza." Madrid, Morata.

Guarro Pallás, A. (2002). "Curriculum y Democracia. Por un cambio de la cultura escolar". Barcelona: Octaedro.

Hoffmann, J. (2010). “Evaluación mediadora” en Anijovich R. (comp.). “La evaluación significativa". Paidós.

Karlheinz Geibler y Hege (1997). “Acción Socioeducativa”. Madrid: Narcea.

Litwin E., y otros. (1998). "La evaluación de los aprendizajes en el debate didáctico contemporáneo". Educador: Paidós.

Pérez Campanero, M. P. (1995). “Como detectar las necesidades de intervención socioeducativa”. Madrid: Narcea.

Perrenoud, P. (1997). “La evaluación de los alumnos. De la producción de la excelencia a la regulación de los aprendizajes. Entre dos lógicas". Colihue. 